\title{
Pflegebildung 2021: Blickpunkt Generalistik
}

\author{
Eine Bestandsaufnahme im Wahljahr Insbesondere im Jahr einer Bundes- \\ tagswahl bietet es sich an, neue politische Rahmenbedingungen und ihre Um- \\ setzung kritisch unter die Lupe zu nehmen. Das gilt auch für die veränderte \\ Ausbildung zukünftiger Pflegefachmänner und -fachfrauen. Was ist gelungen \\ und wo herrscht Verbesserungsbedarf? \& Carsten Drude
}

\section{ZUSAMMENFASSUNG}

\begin{abstract}
In diesem Artikel werden aus Sicht des Bundesverbands Lehrende Gesundheits- und Sozialberufe (BLGS) e.V. einige zentrale und aktuelle Themen der Pflegebildung aufgegriffen und beurteilt.
\end{abstract}

\section{Schlüsselwörter: Bildung, Generalistik, Pflegepädagogen}

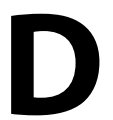
ie Not ist groß - so könnte die pauschale Überschrift über alle Themen und Bereiche der Pflege im Jahr 2021 lauten. Davon sind auch die Pflegeausbildung und die damit verknüpften Nebenschauplätze betroffen. Da der Großteil der Ausbildungsbeginnenden nach wie vor in der zweiten Jahreshälfte startet, ist - zum Erscheinungstermin dieses Artikels - jetzt Halbzeit für die ersten generalistischen Ausbildungskurse. Wie sieht das Zwischenfazit der neuen Ausbildung, die an vielen Stellen unter denkbar ungünstigen, pandemiebedingten Voraussetzungen starten musste, aus?

\section{Zunächst einmal etwas Positives}

Allen Unkenrufen und Weltuntergangsszenarien, die im Rahmen des Gesetzgebungsverfahrens zum Pflegeberufegesetz (PflBG) aufkeimten, zum Trotz, haben die meisten Bildungseinrichtungen und Träger der praktischen Ausbildung einen einigermaßen nahtlosen und verlustfreien Übergang in die neue Ausbildungsform hinbekommen. Die Rückmeldungen unserer Mitglieder und Mitgliedsschulen zeigen auf, dass insbesondere die curriculare Umstellungsarbeit flächendeckend aufgenommen und umgesetzt wurde. Dass es sich dabei um einen Prozess handelt, der nicht zum Stichtag X abgeschlossen sein wird, liegt in der Natur der Sache - curriculare Arbeit lebt von der Evaluation und Verbesserung der Prozesse.
Die Ausbildungsfinanzierung ist durch das in jedem Bundesland eingeführte System der Pauschalbudgets für die Schulen deutlich transparenter und damit planungssicherer geworden. Doch leider schlägt auch hier das nicht immer förderliche föderale System mit aller Macht zu: Die unterschiedlichen Geldbeträge, die pro Ausbildungsplatz pro Jahr ausgeschüttet werden, schwanken von Bundesland zu Bundesland. Das Extrem bietet die Differenz von rund 4.000 Euro zwischen Bayern und Mecklenburg-Vorpommern. Damit wird die Ausbildung nun doch nicht bundesweit vergleichbar - eine Prämisse, die sich alle im Gesetzgebungsverfahren Aktiven auf die Fahnen geschrieben hatten. Als berufspolitischer Erfolg kann die Tatsache gewertet werden, dass der Gesetzgeber im $\$ 14$ (2) der Pflegeberufe Ausbildungs- und Finanzierungversordnung (PflAFinV) grundsätzlich dafür gesorgt hat, dass die Refinanzierung für den theoretischen Bereich der Ausbildung klassen-, beziehungsweise kursweise realisiert wird und nicht tagesgenau abgerechnet werden muss, sollte einmal eine Auszubildende die Ausbildung vorzeitig abbrechen. Aber auch hier zeigt sich, dass dieser Paragraf in den einzelnen Bundesländern unterschiedlich ausgelegt und gehandhabt wird.

Pandemiebedingt war der Ausbildungsbeginn für die angehenden Pflegefachpersonen, wie in allen anderen Ausbildungsberufen auch, unter erschwerten Rahmenbedingungen aber teilweise vom ersten Schultag an, möglich - mit Distanzunterricht und Homeschooling. Erschwerend kommt in unserem Berufszweig hinzu, dass es in vielen Pflegesettings unmittelbar um den Kontakt mit Menschen und deren Bezugspersonen geht - eine Situation, die per Videochat nur sehr schwer zu simulieren ist. Die Umstellung auf digitale Lernangebote musste jedoch zwangsläufig sehr schnell erfolgen, um den Ausbildungsstart und das Ausbildungsziel nicht zu verpassen. Das hat in vielen Schulen hervorragend funktioniert vermutlich hätte dieser Digitalisierungsprozess ohne die zwingende Notwendigkeit in einigen Einrichtungen deutlich länger gedauert. Einziger Wehrmutstropfen: Da sich viele Gesundheitsschulen in Trägerschaft einer größeren Organisation befinden, werden für den Umgang mit den Daten auch die gleichen Maßstäbe wie bei- 
spielsweise für Patienten an den Tag gelegt. Das führte in den vergangenen eineinhalb Jahren des Öfteren dazu, dass erforderliche Software nicht angeschafft oder nicht lizenziert wurde. Hier gilt es, deutlich zu machen, dass es sich bei den schulischen Anforderungen um einen Datenaustausch handelt, der zwingend erforderlich und gewünscht ist.

In der praktischen Ausbildung hat sich gezeigt, dass die vom BLGS prognostizierten Engpässe hinsichtlich der Kapazitäten für die vorgeschriebenen Einsatzorte Schwierigkeiten für die Einsatzplanung zur Folge haben. Insbesondere gilt dies für den Pflichteinsatz in der pädiatrischen Versorgung, aber auch im ambulanten Pflegebereich werden logistische Grenzen gemeldet. Eine flexible Auslegung des PflBG und der dazugehörigen Prüfungsordnung seitens der Behörden wäre hier hilfreich, ohne die Grundintention des generalistischen Gedankens dabei aus den Augen zu verlieren.

\section{Der Kampf um die Spezialabschlüsse}

Im berufspolitischen Alltag auf Ebene von Bund und Ländern wird von einigen Interessenvertreter ${ }^{*}$ innen und Verbänden weiterhin für die Stärkung der im PflBG ebenfalls möglichen separaten Spezialabschlüsse „Altenpflege“ und „Gesundheits- und Kinderkrankenpflege“ gekämpft. Das ist aus jeweiliger Verbandssicht nachvollziehbar, der BLGS bleibt aber bei seiner eindeutigen Empfehlung, ausschließlich die dreijährige generalistische Ausbildung mit dem Abschluss „Pflegefachfrau/Pflegefachmann“ anzubieten. Die Argumente hierzu wurden in den letzten Jahren ausreichend und wohlbegründet veröffentlicht und Rückmeldungen der Auszubildenden bestätigen diese Ausrichtung im positiven Sinne. Ärgerlich ist aus berufspolitischer Sicht die Einmischung fremder Berufsgruppen in diese Diskussion, häufig aus dem ärztlichen Bereich. Man stelle sich nur einmal das Szenario vor, dass die Pflegeverbände bei der nächsten Novelle der ärztlichen Approbationsordnung in ähnlicher Form Vorschriften erlassen und Forderungen stellen würden (by the way: das Medizinstudium ist generalistisch angelegt - die spezialisierte Facharztausbildung kommt erst später). Ich wäre auf den Kommentar der Ärztekammern sehr gespannt.

Die aktuellen Ausbildungszahlen und die damit verknüpften Quoten von Ausbildungsabbrüchen sind noch nicht sehr aussagekräftig, daher ist auch deren Interpretation nur mit Vorsicht vorzunehmen. Zwar hat das statische Bundesamt Ende Juli 2021 erste Daten veröffentlicht, valide Aussagen können aber erst nach dem Abschluss der ersten Kurse getroffen werden. Eigene Befragungen in den Reihen des BLGS e.V. hinsichtlich der Abbruchquoten von Auszubildenden zeigen in der ersten Jahreshälfte 2021 ein breit gestreutes Bild. Teilweise waren die Quoten sehr gering (unter 5\%), teilweise lagen sie bei über einem Viertel der Ausbildungsbeginnenden (27\%). Da diese Befragung eher ein stichtagsbezogenes, mitunter regional gefärbtes Bild wiedergibt und keine Befragung im Sinne einer wissenschaftlich begleitenden Evaluation spiegelt, wurde sie nicht als Fachartikel veröffentlicht. Die in der Arbeitsgruppe 1 der Konzertierten Aktion Pflege (KAP), in der der BLGS als aktives Mitglied auf Bundesebene tätig ist, gesteckten Ziele der Erhöhung der Ausbildungsplätze um 10\% wird vielerorts noch nicht erreicht. Dennoch sind die Bemühungen groß, für den Pflegeberuf zu werben. In einigen Regionen scheint der pandemiebedingte Lockdown dazu geführt zu haben, dass sich junge Menschen

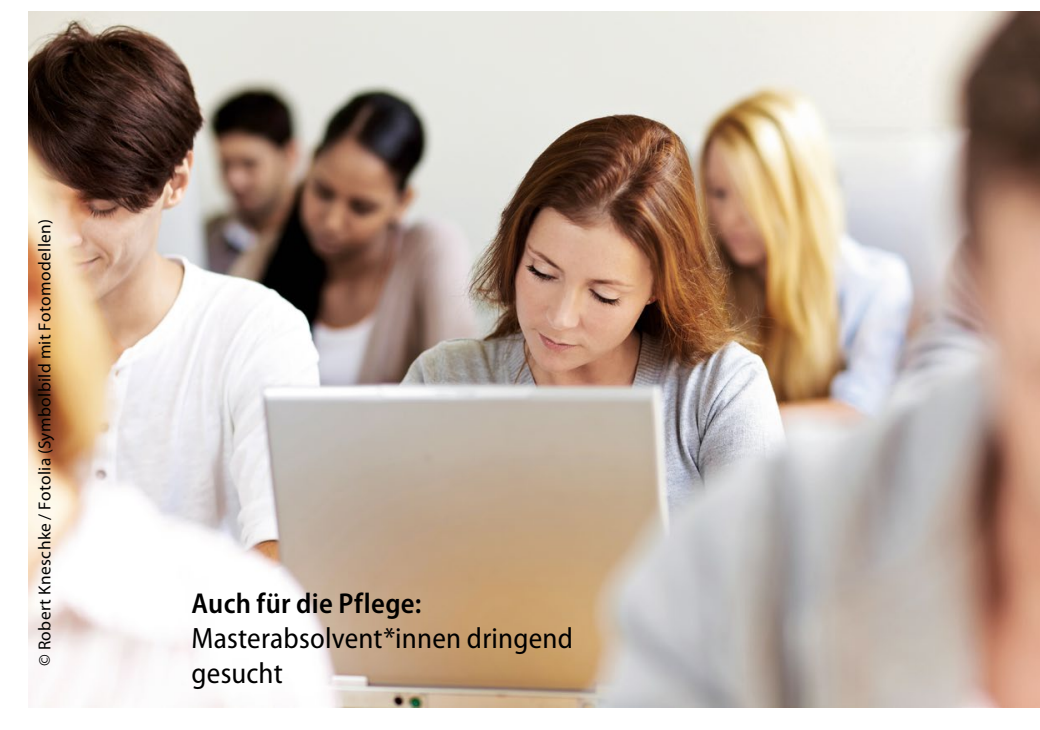

einen sehr krisenfesten Beruf aussuchen, zu denen der Pflegeberuf zweifelsfrei gehört. Dennoch gilt es insbesondere in den praktischen Einsatzorten, die Rahmenbedingungen während der Ausbildung so anzulegen, dass die Pflegefachpersonen nach bestandener Abschlussprüfung gerne wieder dorthin kommen. Das gelingt in Zeiten von flächendeckendem Personalmangel nicht immer.

\section{Wo sind die Lehrer*innen?}

Nicht nur im Bereich der pflegerischen Grundausbildung werden händeringend geeignete Menschen gesucht - der personelle Mangel zeigt sich auch in der Pflegepädagogik. Es gibt schlicht und einfach keine ausreichende Anzahl an Masterabsolventinnen und -absolventen dieses Studienzweigs. Die gesetzlichen Rahmenbedingungen als Vorgabe für Lehrende und Schulen begrüßt der BLGS e.V. sehr - ein bundesweit festgelegtes Verhältnis von Lehrenden zu Lernenden von 1:20 (übrigens in Nordrhein-Westfalen per Verordnung mindestens bis 2025 aufgeweicht) und die hochschulische Qualifikation der Lehrenden auf Masterniveau. Die in Kürze mit dem Renteneintritt beginnende Babyboomer-Generation ist bei dieser Betrachtung des Mangels noch gar nicht berücksichtigt. Der Kampf um geeignete Lehrende wird sich daher verschärfen - erste Schulen melden Kursausfälle zu Ausbildungsbeginn aufgrund von fehlenden Lehrpersonen. Wie kann hier gegengesteuert werden?

Gesteigerte Kapazitäten: Die Anzahl der Studienplätze muss insgesamt deutlich erhöht werden. Es existieren zwar Hochschulen, die damit bereits im Rahmen der Ausbildungsreform begonnen haben, von einem flächendeckenden bundesweiten Angebot sind wir aber immer noch weit entfernt. Noch immer gibt es Bundesländer ohne eine Hochschule mit pflegepädagogischen Studienangeboten. Und dass sich auch im hochschulischen Bereich nur sehr schwer Nachwuchs für Professuren finden lässt, ist ein weiteres, nicht unerhebliches Problem.

Flexible Studienangebote: Die bestehenden Studienangebote der staatlichen Hochschulen müssen nicht nur hinsichtlich der Quan- 
tität ausgebaut, sondern zusätzlich flexibilisiert werden - die positiven Erfahrungen aus dem Digitallernen können als ein Baustein dazu genutzt werden. Teilzeitangebote oder berufsbegleitend ausgerichtete Studiengänge findet man derzeit fast ausschließlich an privaten und damit kostenpflichtigen Hochschulen.

Behördliche Übergangsfristen für Bachelorabsolvent*innen: Das Masterniveau für die Pflegelehre ist unbestritten und richtig, der Weg dorthin kann aber flexibel gestaltet werden. Sofern ein einschlägiger Bachelorabschluss vorliegt, sollten die Absolventen auch für die Lehrtätigkeit an den Pflegebildungseinrichtungen im Rahmen einer vergüteten Anstellung zugelassen werden. Der Umfang der Anstellung und die Dauer des Übergangszeitraums bis zum Vorliegen des Masterabschlusses kann dabei ausgehandelt werden. Gute Erfahrungen unserer Mitgliedsschulen beziffern den Stellenumfang dabei mit durchschnittlich 50\% der Regelarbeitszeit und einem Gesamtzeitraum von maximal fünf Jahren.

Passende Rahmenbedingungen an den Pflegebildungseinrichtungen: Auch in der Bildungseinrichtung vor Ort kann viel für die neuen Kolleg*innen getan werden: Neben den bereits erwähnten Anstellungsangeboten nach Beendigung des Bachelorstudiums für eine definierte Übergangszeit können flexible Arbeitszeitmodelle mit einem großzügigen Arbeitszeitkonto (gemeint ist der genehmigte Zeitraum zum Ausgleich des Stundenkontos) für Studierende interessant sein - im Idealfall bereits als unbefristeter Arbeitsvertrag. Auch die Ermöglichung von bestimmten Arbeitszeitanteilen in Form von Home-Office-Arbeit trägt zur größeren Akzeptanz bei. Einige Schulen haben zudem bereits Förder- und/oder Stipendienprogramme aufgelegt, um auch zur finanziellen Unterstützung beizutragen. Dass ein gutes Begleitprogramm (Coaching/Mentoring/ Einarbeitung) die Basis allen gemeinsamen Arbeitens für den pflegepädagogischen Nachwuchs ist, versteht sich von selbst. Das sollte aber bereits in den Praxisphasen der Studierenden beginnen.

\section{Blickpunkt Praxisanleitung}

Auch vor dem Inkrafttreten des neuen Pflegeberufegesetzes spielten die Praxisanleitenden eine zentrale Rolle im Rahmen der Berufsausbildung. Ihre Bedeutung wurde per Gesetz (PflBG) noch einmal erhöht: Nicht nur die Erhöhung des Stundenumfangs der berufspädagogischen Weiterbildung von 200 auf 300 Stunden Mindestumfang spiegelt das wider - auch die jährlich zu absolvierenden Pflichtfortbildungen mit einem Stundenanteil von immerhin 24 Std./p.a. verdeutlicht die besondere Rolle der Praxisanleitenden.

Wie funktioniert dieses System in den Einrichtungen seit Einführung der generalistischen Ausbildung? Die Rückmeldungen hierzu sind höchst heterogen. Einerseits wurde mehr und mehr erkannt, dass es sich bei den Vorgaben nicht um eine „nice-to-have“Verordnung handelt, sondern um eine Pflichtaufgabe des Gesetzgebers. Seit der zweiten Jahreshälfte 2020 das Thema der Fortbildungen für Praxisanleitende Fahrt auf. Man gewinnt gelegentlich den Eindruck, dass die Einrichtungen den Gesetzestext und die damit verbundenen Verpflichtungen erst relativ spät internalisiert haben. Andererseits mussten sich die Praxisanleitenden binnen kürzester Zeit auf den Zuwachs völlig neuer Auszubildendengruppen einstellen. Die generalistische Ausrichtung des Pflegeberufegeset- zes bringt es mit sich, dass die sektorenübergreifende Rotation von Auszubildenden eine deutliche Steigerung des Arbeitsumfangs impliziert. Wenn dieser Entwicklung nicht in Form von Freistellung für die Anleitungstätigkeit entsprochen wird, ist die Praxisanleitung zum Scheitern verurteilt.

Wie steht es um die Reputation und die Anerkennung von Praxisanleitenden? Ja, sie sind verpflichtender Bestandteil der neuen Pflegeausbildung, führen ihre Aufgaben aber häufig eher mit persönlichem Enthusiasmus als mit formaler, auch monetärer, Anerkennung aus. Erst sehr wenige Tarifwerke haben die Praxisanleitenden fest im Portfolio, in den meisten Einrichtungen ist es vom guten Willen der Unternehmensführung abhängig, ob sowohl Freistellungskontingente als auch eine Höhergruppierung im jeweiligen Entgeltsystem ermöglicht beziehungsweise abgebildet werden.

\section{Weiterbildung für Praxisanleitende als Normalfall?}

Das Anforderungsprofil für Praxisanleitende, insbesondere mit den gestiegenen Aufgaben im Rahmen des PflBG macht deutlich, dass ein pflegepädagogisches Bachelorstudium die angemessene Form der Bildung und der Abschluss für diese Berufsgruppe wäre. Dieses scheint derzeit nicht realisierbar, bleibt aber ein „Fernziel“. Wie sieht es ansonsten mit den Weiterbildungsstrukturen im Gesundheitssystem aus? Die Vielfalt ist groß, staatlich anerkannt ist wenig, es existiert aber in einigen Bereichen eine gewisse einheitliche Systematik. Anerkannte Fachweiterbildungen in der Pflege sind häufig nach einem zweijährigen Strickmuster angelegt. Dabei werden Theoriestundenanteile (720 Std.) mit rotierenden Praxisphasen im jeweiligen Weiterbildungsfeld absolviert. Warum soll das nicht auch für Praxisanleitende möglich sein? Neben einem gewissen $\mathrm{Maß}$ an vereinheitlichten Standards würde damit auch der Grundstein für eine höhere Eingruppierung in den bestehenden Tarifwerken gelegt.

III

\section{Autorenkontakt}

Carsten Drude M.A. ist Geschäftsführer der Franziskus Gesundheitsakademie Münster, Vorsitzender des Bundesverbandes Lehrende Gesundheits- und Sozialberufe e.V. und Lehrbeauftragter der Fachhochschule Münster -

Fachbereich Gesundheit

E-Mail: drude@blgsev.de 